\title{
Correlation effects in the impurity-limited mobility of quantum wires
}

\author{
B. Tanatar \\ Department of Physics, Bilkent University, Bilkent, 06533 Ankara, Turkey \\ A. Gold \\ Laboratoire de Physique des Solides, Université Paul Sabatier, 118 Route de Narbonne, 31062 Toulouse, France
}

(Received 23 November 1994; revised manuscript received 20 March 1995)

\begin{abstract}
We study many-body effects, introduced via the local-field corrections, on the mobility of quasione-dimensional electron systems. The low-temperature mobility due to remote-impurity doping, homogeneous-background doping, interface roughness, and alloy-disorder scattering is calculated using the relaxation time approximation. We find that correlation effects significantly reduce the mobility at low density.
\end{abstract}

\section{INTRODUCTION}

With the recent advances in microfabrication techniques such as molecular-beam epitaxy and lithographic methods, it has been possible to realize quasi-onedimensional (Q1D) electron systems in semiconductors. In these structures, electrons are confined to a region with dimensions of the order of the de Broglie wavelength, but otherwise free to move in one space direction. Since only a limited number of final states are available during the scattering process, the mobility of Q1D electron systems are considerably enhanced, making them potentially important for high-speed device applications.

The ground-state properties and collective excitations in Q1D systems attracted early attention, ${ }^{1}$ and continues $^{2,3}$ to be of interest as new applications such as quantum wire lasers begin to emerge. Mobility limits for charged-impurity scattering in Q1D systems have been calculated by Sakaki, ${ }^{4}$ Fishman, ${ }^{5}$ Lee and Spector, ${ }^{6}$ and Gold and Ghazali ${ }^{7}$ among others within various approximations. Numerical results for the mobility of Q1D systems also appeared. ${ }^{8}$ In particular, Gold and Ghazali $^{7}$ have developed analytical expressions for the Coulomb and electron-impurity interaction potentials using a model of cylindrical quantum wires. Based on the results of Gold and Ghazali, ${ }^{7}$ and extending their results, the mobility in Q1D systems in an axial magnetic field was calculated by Tanatar and Constantinou. ${ }^{9}$

Screening effects have been known to play an important role in the low-dimensional electronic structures. They stem from the many-body interactions and are usually taken into account in the random-phase approximation (RPA). The main purpose of this paper is to study the effects of local-field corrections (which are neglected in the RPA) on the mobility of quasi-one-dimensional systems. We assume that the electrons in the quantum wire are in their lowest subband, as evidenced in the experiments of Gõni et al., ${ }^{2,3}$ and investigate the density dependence of the mobility for different scattering mechanisms such as remote-impurity doping, homogeneous- background doping, interface-roughness scattering, and alloy-disorder scattering. The dielectric function describing the screening of electrons is calculated at finite temperature. Exchange and correlation effects beyond the RPA are taken into account through the local-field correction. Many-body effects in the mobility of Q1D systems were already considered by Gold and Ghazali ${ }^{7}$ in an approximate way. It was argued that many-body effects reduce the mobility. However, in Ref. 7 only the exchange effects were taken into account. In this paper, we incorporate exchange and correlation effects, and show that correlation effects are very important.

The rest of this paper is organized as follows. In Sec. II we introduce the formalism to calculate the mobility, and we discuss its modifications in an approximation, which goes beyond the RPA. The local-field correction is considered in various approximations. In Sec. III we show the effects of the local-field correction on the impuritylimited mobility of Q1D GaAs system. We conclude with a brief summary in Sec. IV.

\section{THEORY}

Gold and Götze ${ }^{10}$ have developed a self-consistent current-relaxation theory to treat the electron dynamics in low-dimensional semiconductor structures. In this approach, the relaxation time $\tau(\omega=0)$, is given for onedimensional systems by ${ }^{7}$

$$
\frac{1}{\tau(\omega=0)}=\frac{k_{F}}{\epsilon_{F}} \frac{\left\langle\left|U\left(2 k_{F}\right)\right|^{2}\right\rangle}{\varepsilon_{t}\left(2 k_{F}\right)^{2}}
$$

from which the mobility may be calculated using $\mu=$ $e \tau(0) / m$. The averaged squared Fourier transform of the random potential $\left\langle|U(q)|^{2}\right\rangle$, describing the electronimpurity scattering, depends on the specific type of impurity, and will be discussed later. $\varepsilon_{t}(q)$ is a screening function related to the static dielectric function $\varepsilon(q)$. It 
is expressed as $\varepsilon_{t}(q)=1+V(q)[1-G(q)] \chi_{0}(q)$, where $\chi_{0}$ is the Lindhard susceptibility, and $G(q)$ is the localfield factor. We note that the relaxation time is given in terms of the susceptibility $\chi(q)$, of the interacting electron gas, ${ }^{10}$ viz., $\left.\chi(q)=\chi_{0}(q) /\left\{1+V(q)[1-G(q)] \chi_{0}(q)\right]\right\}$ with $\chi_{0}(q)$ the free-electron susceptibility. The term $\left\{1+V(q)[1-G(q)] \chi_{0}(q)\right\}$ can be described as a factor $\varepsilon_{t}(q)$, however, $\varepsilon_{t}(q)$ is not the real dielectric function $\varepsilon(q)$, which is given by $1 / \varepsilon(q)=1-V(q) \chi(q)$. Therefore, to indicate the dielectric function for transport properties, we used the symbol $\varepsilon_{t}(q)$.
In Q1D systems $\chi_{0}$, and consequently $\varepsilon_{t}$, diverges at $q=2 k_{F}$ for zero temperature, signaling the Peierls instability. ${ }^{6}$ This would result in an unphysical infinity for the mobility. To circumvent the divergence, the temperature dependence of $\varepsilon_{t}(q)$ needs to be considered. The temperature-dependent dielectric function may be obtained by integrating over the chemical-potentialdependent dielectric function at zero temperature. ${ }^{11}$ An analytical expression for the temperature-dependent dielectric function in the RPA valid for $\epsilon_{F} \gg T$ is given by

$$
\varepsilon_{t}\left(q=2 k_{F}, T \ll \epsilon_{F}\right)=1+\frac{2}{\pi k_{F} a_{B}} \frac{\epsilon_{0}}{2 e^{2}} V\left(2 k_{F}\right)\left[1-G\left(2 k_{F}\right)\right] \ln \left(\frac{8 e^{\gamma}}{\pi} \frac{\epsilon_{F}}{k_{B} T}\right),
$$

where $\gamma=0.577215 \ldots$ is the Euler constant.

In the above formulation, the local-field factor $G(q)$ accounts for the many-body correlations. Taking $G=0$ amounts to the usual random-phase approximation. Disorder effects will also modify the dielectric function, but to lowest order in the impurity density $N_{i}$, our expression, which is based on the Born approximation, remains valid.

For the model of the Q1D system, we take a circular cylinder of radius $R$, and confine the motion of electrons within the cylinder by an infinite potential barrier. Employing the effective mass approximation, the wave functions and energy levels involving the Bessel functions $J_{n}(x)$ and their zeros are obtained in a straightforward manner. In order to get analytical results for the Coulomb and electron-impurity scattering matrix elements, Gold and Ghazali ${ }^{7}$ have proposed approximate expressions for the two lowest subbands in the infinite-barrier height model. The Coulomb interaction between the charge carriers in the lowest subband, using the approximate analytic wave functions, is given by $^{7}$

$$
V(q)=\left(\frac{2 e^{2}}{\epsilon_{0}}\right) \frac{36}{(q R)^{2}}\left[\frac{1}{10}-\frac{2}{3(q R)^{2}}+\frac{32}{2(q R)^{4}}-\frac{64}{(q R)^{4}} I_{3}(q R) K_{3}(q R)\right],
$$

in which $I_{3}(x)$ and $K_{3}(x)$ are the modified Bessel functions of the first and second kind, respectively. Similarly, the electron-impurity interaction potential (for electrons in their lowest subband) is ${ }^{7}$

$$
V_{\mathrm{imp}}(q)=-\left(\frac{2 e^{2}}{\epsilon_{0}}\right) \frac{48}{(q R)^{2}} \times\left\{\begin{array}{l}
\frac{1}{8}-\frac{1}{4}\left(\frac{R_{i}}{R}\right)^{2}+\frac{1}{8}\left[\left(\frac{R_{i}}{R}\right)^{2}+\frac{8}{(q R)^{2}}\right]^{2}-\frac{1}{(q R)^{2}}-\frac{I_{0}\left(q R_{i}\right) K_{3}(q R)}{q R}, \quad R_{i}<R \\
\frac{K_{0}\left(q R_{i}\right) I_{3}(q R)}{q R}, \quad R_{i} \geq R
\end{array}\right.
$$

In the above expression, $R$ and $R_{i}$ denote the radius of the wire and the position of impurity, respectively. We shall use the Coulomb interaction in the calculation of $\varepsilon_{t}\left(2 k_{F}, T\right)$, and the electron-impurity interaction in various models of impurity scattering.

For illustrative purposes, we consider a Q1D GaAs system, with background dielectric constant $\epsilon_{0}=12.9$. The length and energy scales are expressed in terms of the effective Bohr radius, $a_{B}=\epsilon_{0} / m e^{2}$, and effective rydberg $\mathrm{Ry}=\hbar^{2} /\left(2 m a_{B}^{2}\right)$, where $m$ is the effective band mass of electrons in GaAs.

The local-field factor ${ }^{12} G(q)$, involving the properties of uniform electron gas, may be obtained within the approximation scheme of Singwi et al. ${ }^{13}$ (STLS). In one dimension, one finds ${ }^{1}$

$$
G(q)=-\frac{1}{N} \int_{-\infty}^{\infty} \frac{d k}{2 \pi} \frac{k V(k)}{q V(q)}[S(q-k)-1]
$$

and if one uses $S_{\mathrm{HF}}(q)$ in Eq. (5), one obtains the local-field factor in the Hartree-Fock (HF) approxi- mation. $G_{\mathrm{HF}}(q)$ differs somewhat from the Hubbard approximation $^{7}$

$$
G_{H}(q)=\frac{1}{2} \frac{V\left(\sqrt{q^{2}+k_{F}^{2}}\right)}{V(q)}
$$

where the exchange effects are taken into account in a certain way in order to get an analytical expression for the local-field factor. Recently, Gold and Calmels ${ }^{14}$ have developed a sum-rule version of the STLS approach in the two-dimensional (2D) and 3D electron gas to obtain analytical expressions for the local-field factor. A similar analysis $^{15}$ applied to Q1D systems yields the generalized approximation (GA)

$$
G_{\mathrm{GA}}(q)=\frac{1}{2 \pi N R} \frac{1}{C_{21}} \frac{V\left(\sqrt{q^{2}+q_{0}^{2} / C_{11}^{2}}\right)}{V(q)}
$$

where $C_{11}$ and $C_{21}$ are tabulated parameters that depend on the electron density $N$ and wire radius $R$. Here $q_{0}=2 / a_{B} \sqrt{r_{s}}$, where $r_{s}=1 /\left(2 N a_{B}\right)$. Correlation and 


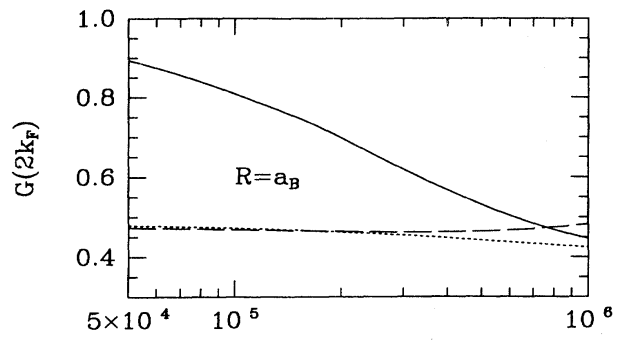

FIG. 1. The local-field factor $G\left(2 k_{F}\right)$ in the generalized approximation (solid line), the HF approximation (dashed line), and the Hubbard approximation (dotted line) as a function of electron density $N$.

exchange effects are included in $G_{\mathrm{GA}}(q)$. For $r_{s}=1$ and $R=a_{B}$ the parameters $C_{i 1}$ are $C_{11}=1.59$ and $C_{21}=0.452$.

In Fig. 1 we compare various approximations to the local-field factor at $2 k_{F}$. The solid line represents $G_{\mathrm{GA}}\left(2 k_{F}\right)$, which is close to $G \approx 1$ for low densities. We observe that $G_{\mathrm{HF}}\left(2 k_{F}\right)$ (dashed line) only differs from the approximate expression (dotted line) beyond $N=10^{6} \mathrm{~cm}^{-1}$. The difference between the exact and approximate forms of $G_{H}$ reaches $\sim 10 \%$ for $N \sim 10^{6} \mathrm{~cm}^{-1}$. The generalized approximation to the local-field factor is markedly different from the Hubbard approximation leading to enhanced reduction of the mobility. This indicates that correlation effects are very important at low densities, i.e., $N<5 \times 10^{5} \mathrm{~cm}^{-1}$. In the following calculations of the impurity-limited mobility we use the parametrized analytical expressions ${ }^{15}$ of the generalized approximation $G_{\mathrm{GA}}\left(2 k_{F}\right)$ to study the effects of localfield corrections, and compare our results with the RPA $(G=0)$.

\section{RESULTS AND DISCUSSION}

We now employ the static dielectric function including the local-field corrections in the calculation of impuritylimited mobility. For the scattering by remote impurities, we assume that the impurities are located randomly at some distance $R_{i}$ from the axis of the quantum wire of radius $R$, with the random potential taken as $\left\langle|U(q)|_{\mathrm{RD}}^{2}\right\rangle=$ $N_{i}\left[V_{\text {imp }}\right]^{2}$. Here $N_{i}$ is the (one-dimensional) impurity density, and $V_{\text {imp }}(q)$ has to be evaluated at the impurity positions $R$. The mobility for remote doping takes the form

$$
\mu_{\mathrm{RD}}=\left(\frac{e a_{B}^{2}}{\hbar}\right) \frac{\pi}{16} \frac{N}{N_{i}} \frac{\left[\varepsilon_{t}\left(2 k_{F}, T\right)\right]^{2}}{\left[F_{\mathrm{imp}}\left(2 k_{F}\right)\right]^{2}},
$$

where we used $V_{\mathrm{imp}}=\left(2 e^{2} / \epsilon_{0}\right) F_{\mathrm{imp}}$. In order to assess the importance of local-field corrections, we display in Fig. 2 the mobility due to remote-impurity doping for a wire radius $R=a_{B}$ and impurities located on the wire axis $\left(R_{i}=0\right)$. Shown by solid, dashed, and dot-dashed lines are the mobility results calculated with $G_{\mathrm{GA}}, G=0$ (which corresponds to the RPA), and $G_{H}$, respectively.

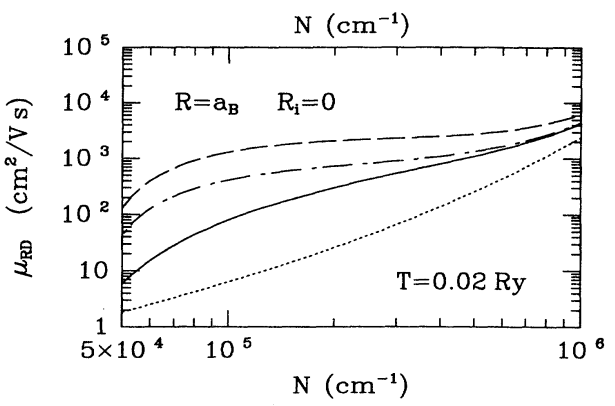

FIG. 2. Mobility for remote doping as a function of electron density for $R=a_{B}$ and $R_{i}=0$. Solid, dashed, and dot-dashed lines are calculated with the local-field factors $G_{\mathrm{GA}}, G=0$ (RPA), and $G_{H}$, respectively. The dotted line represents the unscreened limit $\left(\varepsilon_{t}=1\right)$.

The dotted line represents the unscreened limit of $\mu_{\mathrm{RD}}$ for which $\varepsilon_{t}=1$. We observe that the mobility calculated with $G_{\mathrm{GA}}(q)$ approaches the unscreened limit as the electron density $N$ decreases. This is a consequence of the fact that the strong correlation regime corresponds to the unscreened limit in Q1D electron systems.

In Fig. 3 we show the mobility due to remote-impurity doping with (solid lines) and without (dashed lines) the local-field correction $\left(G_{\mathrm{GA}}\right)$ for various impurity locations. The parameters used are $N_{i}=10^{6} \mathrm{~cm}^{-1}, R=a_{B}$, and $T=0.02 \mathrm{Ry}$. The striking observation is that the local-field corrections decrease the mobility substantially in the low density region (viz., $N<5 \times 10^{5} \mathrm{~cm}^{-1}$ ). As $N$ increases, the effects of local-field corrections subside and the mobility approaches the RPA limit. For the dotted lines in Fig. 2 we have included the the local-field corrections in the HF approximation. The difference between the solid lines and the dotted lines is because of correlation effects. We conclude that not only exchange effects reduce the mobility, ${ }^{7}$ but also correlation effects considerably reduce the mobility in one-dimensional systems at low density. In Fig. 4 we study the mobility for remote doping for different wire radii and $R_{i}=2 R$. With increasing wire radius the mobility increases strongly and many-body effects are stronger in thinner wires.

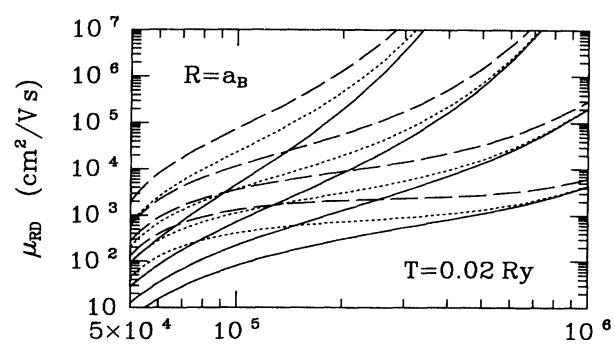

FIG. 3. Mobility for remote doping as a function of electron density. The solid and dashed lines represent calculations with and without local-field corrections, respectively. Curves from bottom to top are for $R_{i}=0,1,2$, and $4 R$. The dotted lines correspond to the HF approximation. 


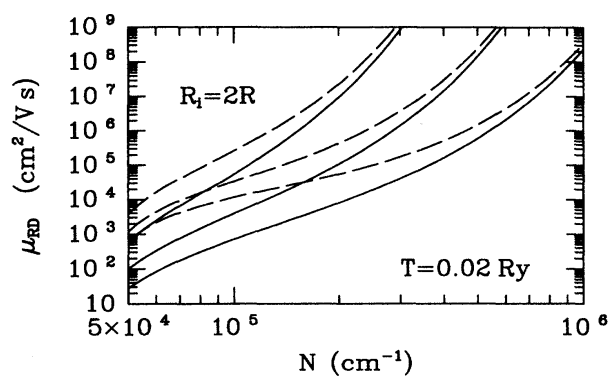

FIG. 4. Mobility for remote doping as a function of electron density and $R_{i}=2 R$. The solid and dashed lines represent calculations with and without local-field corrections, respectively. Curves from bottom to top are for $R=a_{B}, 2 a_{B}$, and $4 a_{B}$. The impurity density is $N_{i}=10^{6} \mathrm{~cm}^{-1}$.

In order to describe impurities introduced by homogeneous-background doping, we assume the impurities are homogeneously distributed within the wire $\left(0<R_{i}<R\right)$. The random potential in this case is defined as ${ }^{7}$

$$
\left\langle|U(q)|^{2}\right\rangle=N_{B} \int_{0}^{R} d r r\left|V_{\mathrm{imp}}(q)\right|^{2},
$$

where $N_{B}$ is the $3 \mathrm{D}$ impurity density. Defining $\left\langle|U(q)|^{2}\right\rangle=N_{B} R^{2}\left(2 e^{2} / \epsilon_{0}\right)^{2} F_{B}$, where the explicit form of $F_{B}(q)$ is given by Gold and Ghazali, ${ }^{7}$ the mobility for homogeneous-background doping becomes

$$
\mu_{\mathrm{BD}}=\left(\frac{e a_{B}^{2}}{\hbar}\right) \frac{\pi N}{N_{B} R^{2}} \frac{\left[\varepsilon_{t}\left(2 k_{F}, T\right)\right]^{2}}{F_{B}\left(2 k_{F}\right)}
$$

We show the mobility for the above model as a function of electron density in Fig. 5. For charge neutrality in uncompensated semiconductors, we take $N_{B} R^{2} / 2=N$. Shown in Fig. 5 is the mobility for wire radii $R=a_{B}$, $2 a_{B}$, and $4 a_{B}$ (from bottom to top) with and without the local-field corrections, indicated by solid and dashed curves, respectively.

Interface-roughness scattering is known to be the dominant scattering mechanism for $2 \mathrm{D}$ electron gas in thin quantum wells. Assuming a Gaussian-like decay of

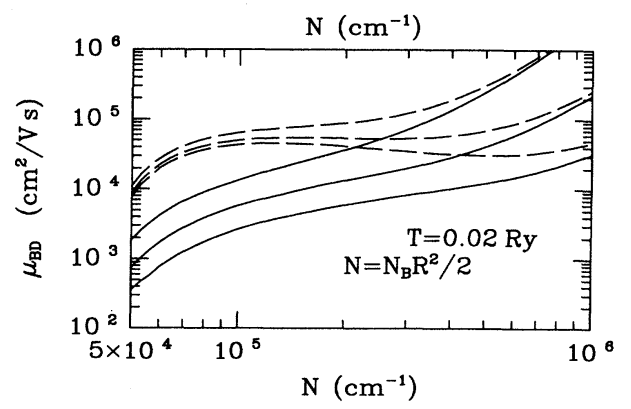

FIG. 5. Mobility for homogeneous-background doping as a function of electron density. The solid and dashed lines represent calculations with and without local-field corrections, respectively. Curves from bottom to top are for $R=1,2$, and $4 a_{B}$.

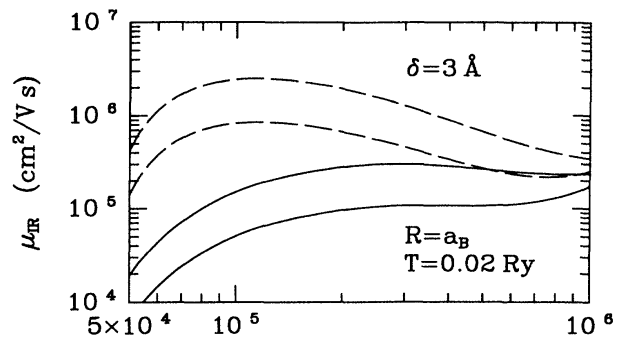

FIG. 6. Mobility for interface-roughness scattering as a function of electron density. The solid and dashed lines represent calculations with and without local-field corrections, respectively. Top and bottom curves are for $\eta=20$ and 60 $\AA$, respectively.

the roughness fluctuations, and following the example of $2 \mathrm{D}$ systems, ${ }^{16}$ the random potential is obtained as $\left\langle|U(q)|^{2}\right\rangle=\left(d E_{01} / d R\right)^{2} \eta \delta^{2} \pi^{1 / 2} e^{-q^{2} \eta^{2} / 4}$, where $\delta$ and $\eta$ are the height and range parameters. One gets for the interface-roughness mobility ${ }^{7}$

$$
\mu_{\mathrm{IR}}=\left(\frac{e a_{B}^{2}}{\hbar}\right) \frac{\pi^{1 / 2}}{4} \frac{R^{6} N e^{k_{F}^{2} \eta^{2}}}{\beta_{01}^{4} \eta \delta^{2} a_{B}^{2}}\left[\varepsilon_{t}\left(2 k_{F}, T\right)\right]^{2} .
$$

The mobility for interface-roughness scattering as a function of the electron density for a GaAs quantum wire of radius $R=a_{B}$ and average roughness size $\delta=3 \AA$ are shown in Fig. 6. Solid and dashed lines indicate calculations with and without local-field corrections, respectively, for two different values of the range parameter $\eta=20 \AA$ and $60 \AA$ (upper and lower curves, respectively). The mobility for interface-roughness scattering is strongly reduced for $N<3 \times 10^{5} \mathrm{~cm}^{-1}$ when the localfield correction is included.

The random potential describing the alloy-disorder scattering is $\left\langle|U(q)|^{2}\right\rangle=\left[(\delta V)^{2} a^{3} / 4 a_{B}^{2}\right] x(1-x) F_{\mathrm{AD}}$, in which $\delta V$ is the root-mean-square spatial average of the fluctuating alloy potential over the alloy unit cell, and $a^{3}$ is the unit-cell volume. $F_{\mathrm{AD}}$ is the form factor for the lowest subband, defined as $F_{\mathrm{AD}}=a_{B}^{2} \int d^{2} \mathbf{r}|\phi(\mathbf{r})|^{4}$, which yields $F_{\mathrm{AD}}=9 a_{B}^{2} /\left(5 \pi R^{2}\right)$ when the approximate wave function for the lowest subband is used. The mobility

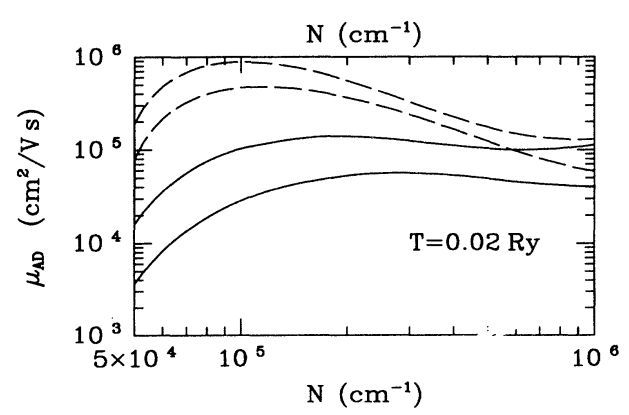

FIG. 7. Mobility for alloy-disorder scattering as a function of electron density for $x=0.3$. The solid and dashed lines represent calculations with and without local-field corrections, respectively. Lower and upper curves correspond to $R=a_{B}$ and $2 a_{B}$, respectively. 
due to alloy-disorder scattering takes the form ${ }^{17}$

$$
\mu_{\mathrm{AD}}=\left(\frac{e a_{B}^{2}}{\hbar}\right) 4 \pi N a_{B}\left(\frac{a_{B}}{a}\right)^{3} \frac{\left[\varepsilon_{t}\left(2 k_{F}, T\right)\right]^{2}}{(\delta V)^{2} x(1-x) F_{\mathrm{AD}}}
$$

in which $\delta V$ is expressed in rydbergs. In Fig. 7 we show the dependence of the mobility due to alloy-disorder scattering on the electron density. Solid and dashed lines indicate calculations with and without local-field corrections, respectively, at $T=0.02 \mathrm{Ry}, x=0.3, a=5.66 \AA$, and $\delta V \simeq 1 \mathrm{eV}$. Lower and upper curves correspond to $R=a_{B}$ and $2 a_{B}$, respectively, illustrating the $R$ dependence of the mobility.

\section{CONCLUSION}

We have studied the influence of many-body effects on the impurity-limited mobility of a Q1D electron system. The local-field correction is used in the generalized approximation taking into account exchange and correlation. In contrast to the usually employed RPA, local-field corrections significantly reduce the mobility at low densities. This general trend is found in the calculations of remote doping, homogeneous-background doping, interface-roughness scattering, and alloy-disorder scattering. In this paper, we have mainly focused on the density dependence of the mobility. More systematic studies of the dependence on the wire radius could be undertaken as new experimental results become available. The results presented in this paper are for low temperatures, but extension to higher temperatures and a systematic study of the temperature dependence of the mobility is also possible. Our results indicate that transport calculations neglecting many-body effects cannot predict the order of magnitude of the mobility.

\section{ACKNOWLEDGMENTS}

This work is supported in part by the Scientific and Technical Research Council of Turkey (TUBITAK). B.T. thanks A. Kürkçüoğlu for useful discussions, and Dr. N. C. Constantinou for valuable comments. The Laboratoire de Physique des Solides (URA74) is a Laboratoire associé au Centre National de la Recherche Scientifique (CNRS).
${ }^{1}$ W. I. Friesen and B. Bergersen, J. Phys. C 13, 6627 (1980).

${ }^{2}$ A. R. Gõni, A. Pinczuk, J. S. Weiner, J. M. Calleja, B. S. Dennis, L. N. Pfeiffer, and K. W. West, Phys. Rev. Lett. 67, 3298 (1991).

${ }^{3}$ A. Schmeller, A. R. Gõni, A. Pinczuk, J. S. Weiner, J. M. Calleja, B. S. Dennis, L. N. Pfeiffer, and K. W. West, Phys. Rev. B 49, 14778 (1994).

${ }^{4}$ H. Sakaki, Jpn. J. Appl. Phys. 19, L735 (1980); J. Vac. Sci. Technol. 19, 148 (1981).

${ }^{5}$ G. Fishman, Phys. Rev. B 34, 2394 (1986).

${ }^{6}$ J. Lee and H. Spector, J. Appl. Phys. 57, 366 (1985); 54, 3921 (1983).

${ }^{7}$ A. Gold and A. Ghazali, Phys. Rev. B 41, 7626 (1990).

${ }^{8}$ Y. Weng and J. P. Leburton, J. Appl. Phys. 65, 3089 (1989).

${ }^{9}$ B. Tanatar and N. C. Constantinou, J. Phys. Condens. Matter 6, 5113 (1994).
${ }^{10}$ A. Gold and W. Götze, Phys. Rev. B 33, 2495 (1986).

${ }^{11}$ P. Maldague, Surf. Sci. 73, 296 (1978); N. R. Arista and W. Brandt, Phys. Rev. A 29, 1471 (1984).

${ }^{12}$ K. S. Singwi and M. P. Tosi, Solid State Phys. 36, 177 (1981).

${ }^{13}$ K. S. Singwi, M. P. Tosi, R. H. Land, and A. Sjölander, Phys. Rev. 176, 589 (1968).

${ }^{14}$ A. Gold and L. Calmels, Phys. Rev. B 48, 11622 (1993).

${ }^{15} \mathrm{~L}$. Calmels and A. Gold, Solid State Commun. 92, 619 (1994); 93, 9i(E) (1995).

${ }^{16}$ T. Ando, A. B. Fowler, and F. Stern, Rev. Mod. Phys. 54, 437 (1982).

${ }^{17}$ A. Gold and A. Ghazali, Solid State Commun. 83, 661 (1992). A factor $\left(a_{B} / a\right)^{3}$ is missing in Eq. (3) of this reference because of a printing error, and $h$ has to be replaced by $\hbar$. 